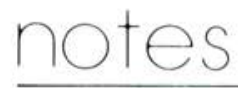

\title{
Dosimétrie des personnes exposées aux radiations dans l'exercice de leur profession en Suisse
}

\author{
Méthodes et résultats \\ 9e rapport de la Commission fédérale de la protection contre \\ les radiations pour l'année 1984, établi par le groupe d'experts \\ pour la dosimétrie*
}

(Manuscrit reçu le 31 juillet 1985)

\section{RESUME}

Cette note présente le bilan dosimétrique des irradiations professionnelles en Suisse pour l'année 1984. Ce document est publié chaque année depuis 1975 par l'Office fédéral de la santé publique (Berne).

\section{ABSTRACT}

The dose records for occupational exposures in Switzerland are presented for the year 1984. The report has been published by the Office fédéral de la santé publique (Federal office for public health) in Bern every year since 1975.

Dans la plupart des pays, la dosimétrie individuelle des personnes professionnellement exposées aux rayonnements ionisants est effectuée par un service central de l'administration gouvernementale. En Suisse, 12 services de dosimétrie homologués font ce travail sous la surveillance de 3 instances de contrôle. Malgré cette décentralisation, qui s'accompagne d'une diversification des méthodes de mesure, un niveau suffisant de standardisation et de cohérence a pu être atteint, grâce à la fonction coordonnatrice du groupe d'experts pour la dosimétrie individuelle. Celuici, entre autres activités, organise chaque année, sur mandat des instances de contrôle, une mesure d'intercomparaison à laquelle les services de dosimétrie homologués sont tenus de participer. On attend de ces services qu'ils mesurent en routine toutes les doses supérieures à 50 mrem avec une précision située entre $+50 \%$ et $-30 \%$, et qu'ils notifient spécialement, le jour même, les irradiations extraordinaires.

En 1984, quelque 50000 personnes ont été suivies par les services de dosimétrie individuelle, et aucune dose n'a dépassé les limites annuelles. Le rapport annuel présente la statistique des doses au corps

* S. Prêtre, président, Division principale de la sécurité des installations nucléaires DSN (HSK), CH 5303 Würenlingen; A. Auf der Maur, A. Donath, J. Dutrannois, H. Lengweiler, H. Lüthy, B. Michaud, Th. Samuel, J.F. Valley, Ch. Wernly, membres. 
entier par irradiation externe, des doses aux mains, à la peau, ainsi que des incorporations de substances radioactives (en particulier de tritium). Les doses au corps entier par irradiation externe dominent : elles représentent environ $95 \%$ de la dose collective totale. Parmi les quelque 50000 personnes soumises au contrôle dosimétrique en 1984, moins de 2000 ont accumulé des doses professionnelles supérieures à l'irradiation naturelle. Depuis le début des relevés statistiques (1976), la dose collective totale des personnes exposées aux rayonnements dans l'exercice de leur profession a tendance à diminuer malgré une augmentation des activités professionnelles. Cette situation est le reflet d'attitudes de plus en plus positives vis-à-vis de la radioprotection.

\section{INTRODUCTION}

L'irradiation des personnes exposées aux rayonnements ionisants dans l'exercice de leur profession est contrôlée par des services de dosimétrie homologués, selon l'ordonnance du 11 novembre 1981 sur l'homologation et l'exploitation de services de dosimétrie individuelle (ordonnance sur la dosimétrie). Le groupe d'experts pour la dosimétrie individuelle en précise les modalités d'application. Ainsi, il a, par exemple, élaboré au cours de l'année une " recommandation touchant au port du dosimètre en cas d'utilisation d'un tablier de protection".

Les services de dosimétrie participent chaque année à une mesure d'intercomparaison, ce qui permet de vérifier la conformité des résultats aux normes fixées par l'ordonnance sur la dosimétrie. Ainsi, la décentralisation et la diversification des services de dosimétrie ne constituent plus un désavantage ; au contraire, elles offrent une possibilité d'adaptation aux techniques et besoins nouveaux.

\section{SERVICES DE DOSIMETRIE INDIVIDUELLE}

La mesure des doses individuelles a été effectuée par les services homologués suivants: Organisation européenne pour la recherche nucléaire (CERN), Genève ; COMET S.A., Liebefeld ; Institut fédéral de recherche en matière de réacteurs EIR, Würenlingen ; Institut de radiophysique appliquée (IRA), Lausanne; Centrale nucléaire de Beznau (KKB), Döttingen ; Centrale nucléaire de Gösgen (KKG), Däniken ; Centrale nucléaire de Leibstadt (KKL), Leibstadt ; Centrale nucléaire de Mühleberg (KKM), Mühleberg ; Prof. Dr. H. Lüthy (LY) Riehen ; Service de dosimétrie individuelle (PEDOS), Bremgarten-Berne; Service cantonal de contrôle des irradiations (SCCI), Genève ; Caisse nationale d'assurance en cas d'accidents (SUVA), Lucerne.

Les méthodes de mesure ainsi que le nombre de personnes professionnellement exposées aux radiations, surveillées par les différents services, sont donnés au tableau I.

\section{MESURES D'INTERCOMPARAISON}

\subsection{Irradiation externe}

La $9^{e}$ intercomparaison concernait l'irradiation externe et son organisation a été confiée à l'Institut de radiophysique appliquée à Lausanne. II s'agissait de vérifier : 
1. que les services de dosimétrie sont à même de mesurer les doses d'irradiation situées entre 50 mrem et 500 rem avec une précision située entre $+50 \%$ et $-30 \%$ en routine ;

2. que, lorsque l'on soupçonne une irradiation extraordinaire, les services notifient les résultats le jour même.

Tous les services homologués ont participé à l'intercomparaison. La précision des résultats correspond aux exigences de l'ordonnance sur la dosimétrie, à l'exception d'un cas dû à un incident d'exploitation.

Les délais de notification ont été respectés, à l'exception d'un cas où les dosimètres n'ont pas été transmis directement au service de dosimétrie. Ceci n'a pas d'incidence pratique, ce service n'effectuant pas de dosimétrie pour des usagers extérieurs.

\subsection{Incorporation}

La $10^{e}$ intercomparaison, facultative, consistait en une mesure d'incorporation de tritium. Son organisation a été confiée au laboratoire de la Commission fédérale de surveillance de la radioactivité à Fribourg. Le but de cette intercomparaison était de déterminer avec quelle précision ces mesures d'activité sont effectuées en fonction de l'activité et de la nature des échantillons. 27 laboratoires, dont certains sont des services de dosimétrie individuelle, ont participé à cette intercomparaison, qui consistait à mesurer 2 échantillons d'urine et 3 échantillons d'eau. L'activité de ces échantillons correspondait à des valeurs généralement rencontrées en pratique. 17 laboratoires ont délivré des résultats conformes à la "recommandation concernant la surveillance d'incorporation" du 3 juin 1982 du groupe d'experts (précision $\pm 20 \%$ ). Une nette amélioration a été observée depuis la première intercomparaison de tritium de mai 1982 et, dans l'ensemble, les résultats sont satisfaisants. II paraît, cependant, nécessaire de renouveler ce genre d'intercomparaison tous les 2 ou 3 ans.

\section{IRRADIATION EXTERNE}

\subsection{Dose au corps entier par irradiation externe}

Le tableau II donne la répartition des personnes par secteur d'activité et par intervalle de dose après déduction de l'irradiation naturelle.

La dose au corps entier par irradiation externe pour les personnes professionnellement exposées aux radiations est limitée à 5 rem par année. Sur les 49950 personnes surveillées durant l'année 1984, aucune n'a dépassé cette limite. La dose collective accumulée en 1984 par ces personnes est de 1682 homme-rem. Cela correspond à une dose individuelle moyenne de $34 \mathrm{mrem}$. Le détail de ces doses collectives est donné dans le tableau III.

\subsection{Doses partielles par irradiation externe}

Six services sont à même d'effectuer les mesures de dose aux mains. Le tableau IV donne, par secteur et par intervalle de dose, la répartition des doses enregistrées. 
La dose aux mains pour les personnes professionnellement exposées aux rayonnements est limitée à 75 rem par année. 702 personnes ont ainsi été surveillées, aucune ne dépasse $30 \%$ de la limite maximale admise.

La dose à la peau pour les personnes professionnellement exposées aux rayonnements est limitée à 30 rem par année. 7 services disposent de dosimètres capables d'effectuer ces mesures. La contribution des rayonnements de basse énergie étant faible, les doses à la peau ne dépassent guère les doses au corps entier.

\section{INCORPORATION}

L'estimation de la dose due à l'incorporation s'effectue indirectement par la détermination de l'activité présente dans certains organes ou dans tout l'organisme. La mesure de l'activité présente dans certains organes peut se faire à l'aide d'un compteur externe, comme pour la mesure du radioiode dans la thyroïde, par exemple. La détermination de l'activité présente dans tout l'organisme peut s'effectuer soit avec un anthropogammamètre (compteur corps entier), soit par la mesure de l'activité dans les excrétions.

Les résultats de ces mesures sont donnés dans le tableau $\mathrm{V}$ pour les divers secteurs d'activité en fonction des pourcentages de la limite annuelle d'incorporation. 2519 personnes ont été contrôlées en 1984 : on n'a constaté aucun dépassement des valeurs maximales admissibles. En cas d'incorporation supérieure à $2 \%$ de cette limite, les nucléides incorporés sont mentionnés. II s'agit le plus souvent de tritium. Les doses totales dues à l'incorporation ne représentent que $5 \%$ des doses externes. Cependant, dans le cas du tritium, elles sont importantes pour un petit groupe de personnes.

\section{DEPASSEMENTS DES LIMITES ANNUELLES}

En 1984, aucun dépassement des limites annuelles n'a été enregistré.

\section{CONCLUSIONS}

Au cours de ces dernières années, le nombre de personnes faisant l'objet d'une surveillance dosimétrique a régulièrement augmenté, alors que la dose collective a tendance à diminuer dans tous les secteurs d'activité. 1984 apparaît comme une année particulière :

- aucune dose ne dépasse les limites annuelles,

- la dose collective totale est remarquablement basse.

II est intéressant de constater que sur les quelque 50000 personnes contrôlées, 47400 ont reçu une dose professionnelle annuelle inférieure à 100 mrem, soit moins que l'irradiation naturelle.

Nous remercions sincèrement ici $M$. H. Felber (OFSP), qui a rédigé les procès-verbaux des séances et accompli de nombreux travaux d'organisation. M. H.J. Pfeiffer (DSN) a assisté le président du groupe d'experts dans différentes tâches, tout en remplissant les fonctions de secrétaire scientifique. Le présent rapport a également bénéficié de la collaboration active de M. J.C. Corminboeuf (SCCI). Qu'ils trouvent ici nos remerciements cordiaux. 
NOTES

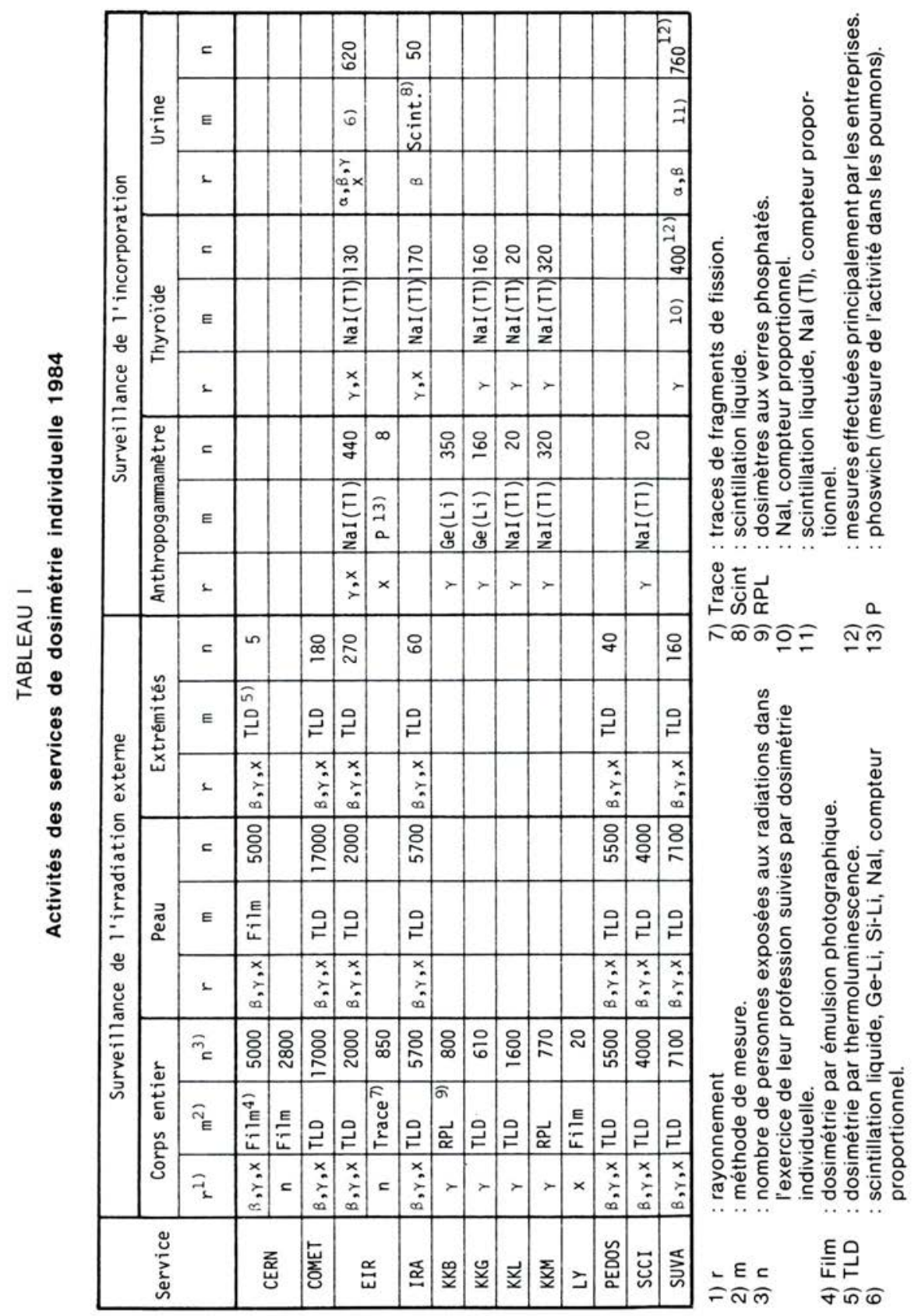


NOTES

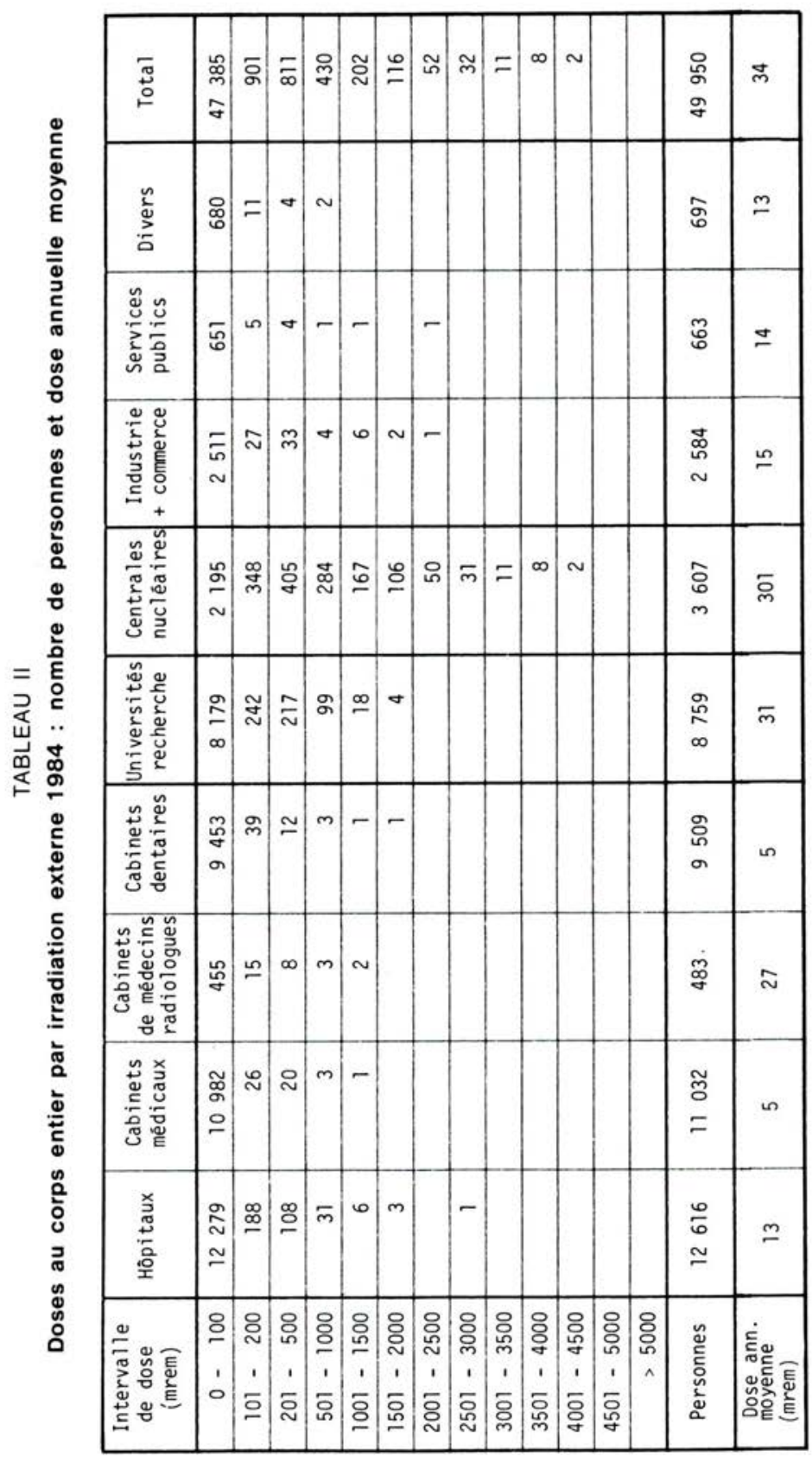


NOTES

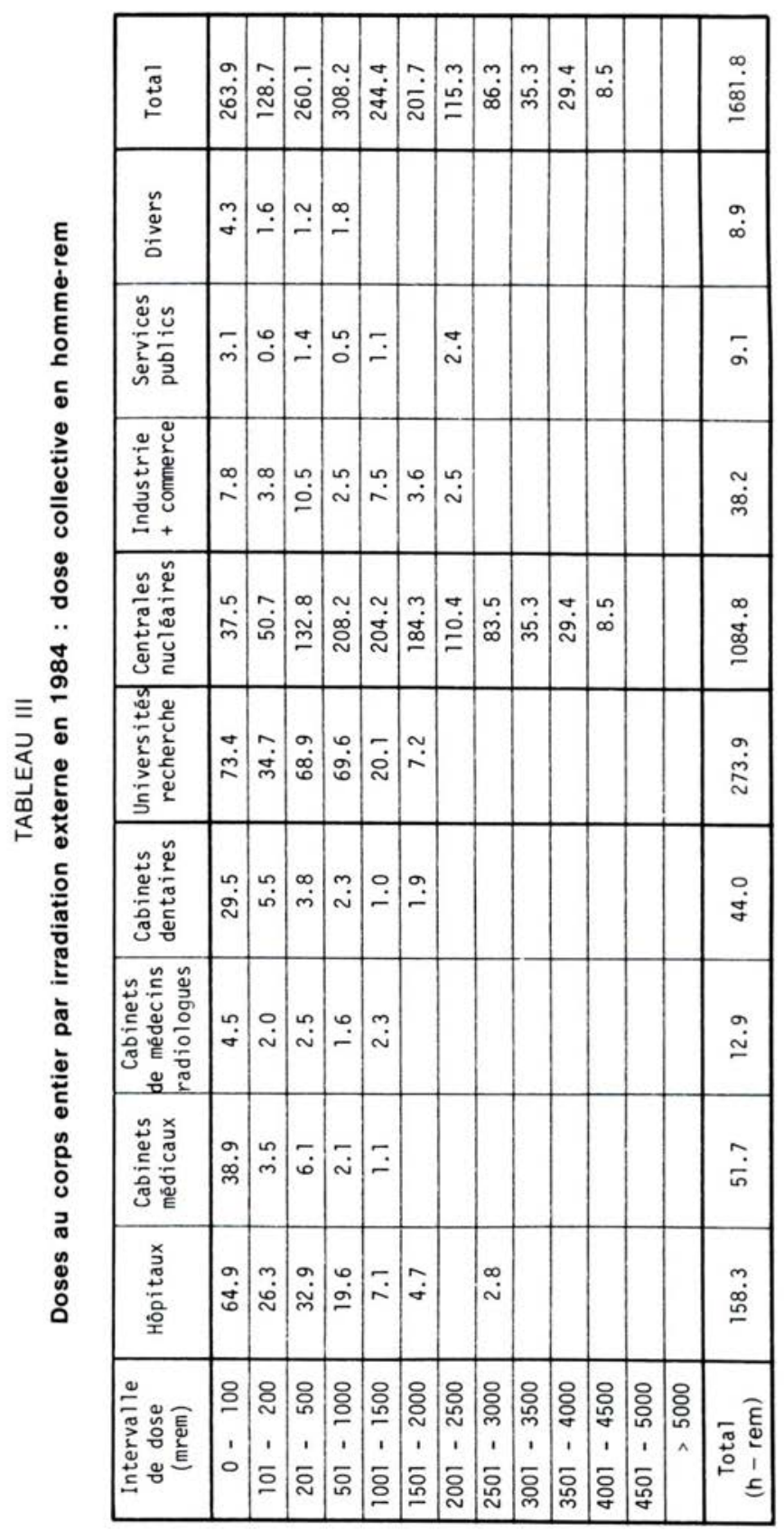


NOTES

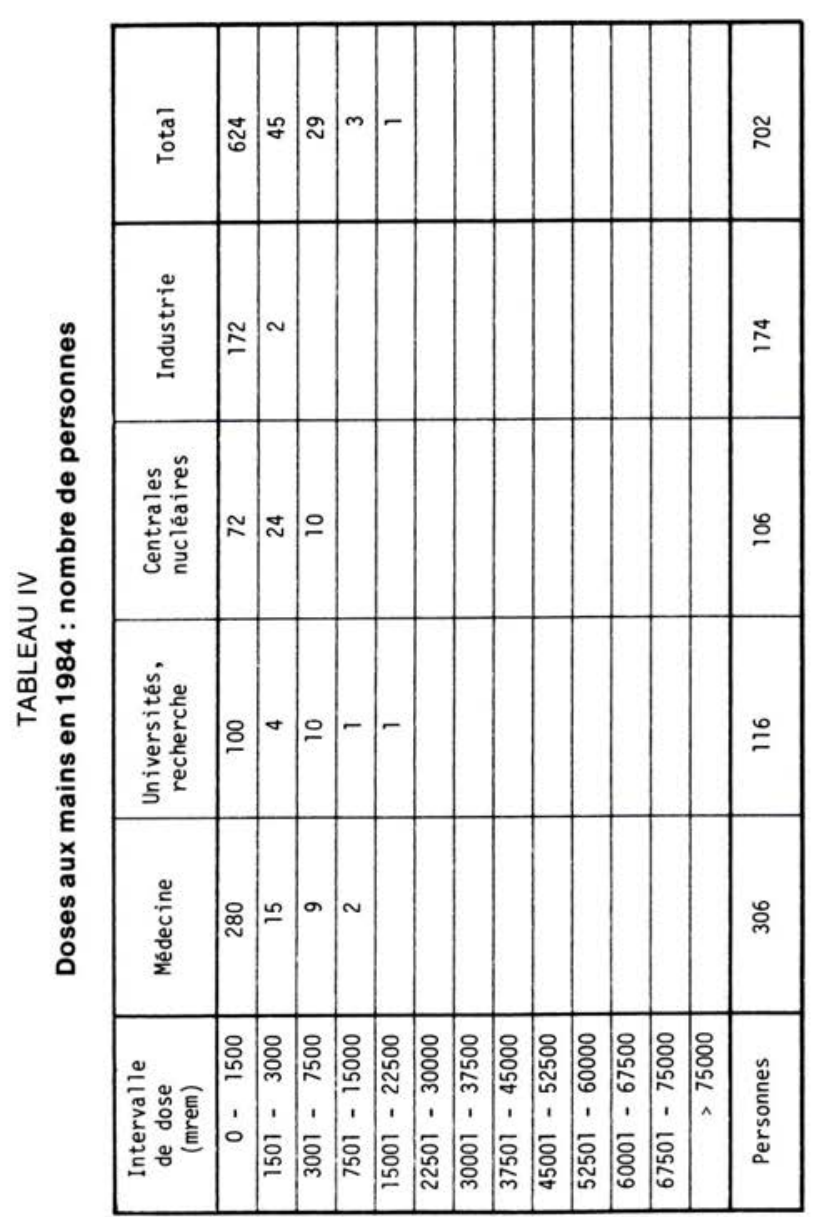




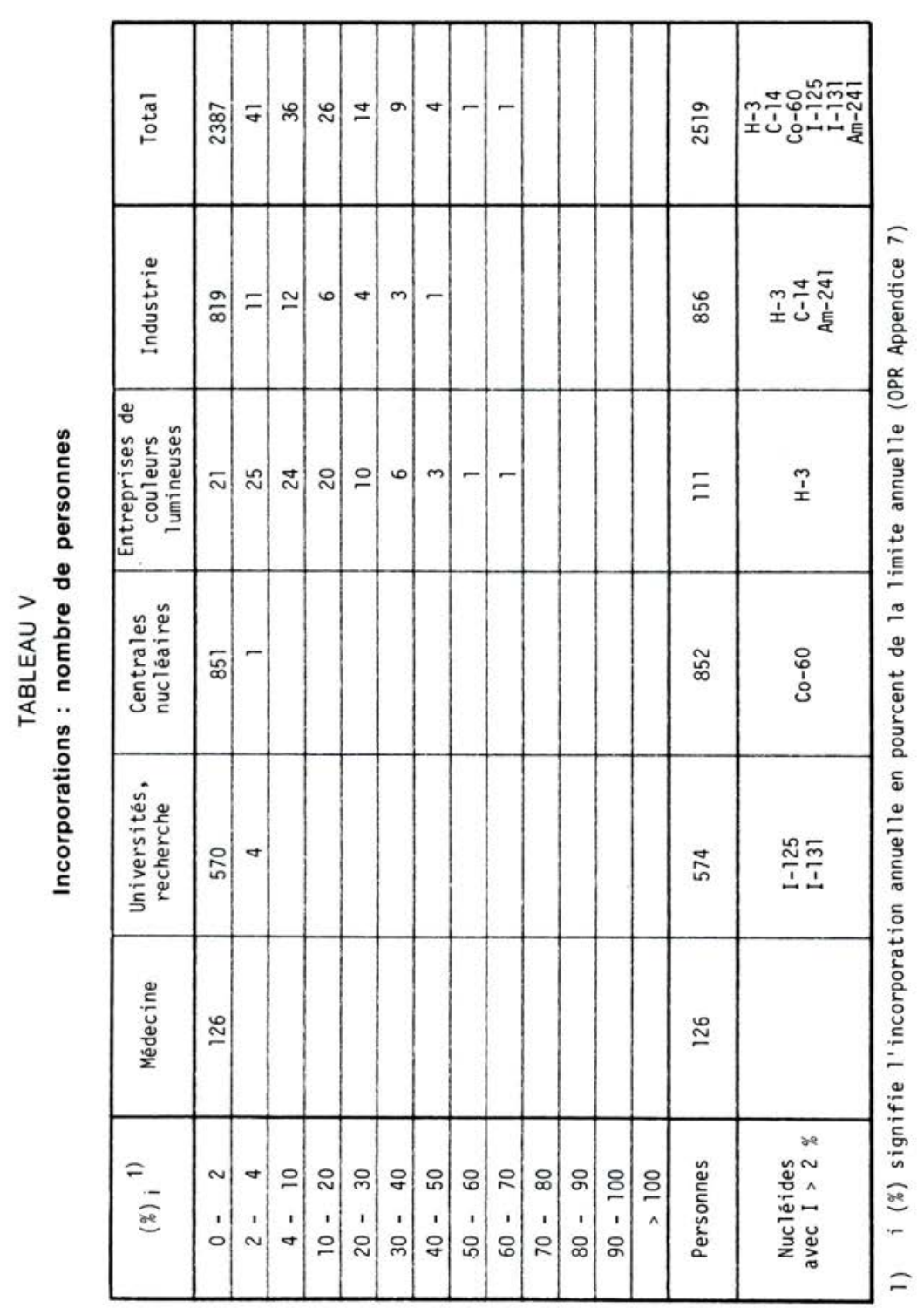

